\title{
Thomas, Martin/Thompson, Andrew S. (Hrsg.): The Oxford Handbook of the Ends of Empire, 800 S., Oxford UP, Oxford u. a. 2018.
}

\author{
Jürgen Osterhammel
}

Online publiziert: 17. Januar 2020

(C) Der/die Autor(en) 2019

Handbooks - oft weniger systematisch angelegt als „Handbücher“ in der deutschen Tradition - und Companions über historische und sozialwissenschaftliche Themen erfreuen sich berechtigter Beliebtheit. Im Idealfall kartieren sie ein Gebiet, führen die besten Expertinnen und Experten zusammen, registrieren die maßgebende Literatur, geben Impulse für künftige Forschungen und vermitteln im akademischen Unterricht Grundkenntnisse und Diskussionsanregungen. Nicht alle Felder sind jedoch in gleichem Maße handbuchtauglich. Manche Handbooks bleiben allzu programmatisch oder bilden doch nur eine bestimmte Forschungsrichtung ab, andere sind so bieder und konventionell, dass sie für Kenner der Materie kaum Überraschendes bieten.

Keiner dieser Kritikpunkte kann an das „Oxford Handbook of the Ends of Empire“ gerichtet werden. „Ends of Empire“ meint hier die Dekolonisation - dieser Begriff erscheint auch in sehr vielen Überschriften der 37 Kapitel - nach dem Zweiten Weltkrieg mit leichter zeitlicher Erweiterung zurück bis 1918 und räumlicher Ergänzung durch Gebiete, an die man bei „Entkolonialisierung“ seltener denkt, etwa China, Osteuropa oder das, was Robert S.G. Fletcher in einem der originellsten Kapitel des Buches ,the arid world“ nennt, also „the world's desert environments“ (S. 373). Nicht behandelt werden aus guten praktischen Gründen Reichszusammenbrüche in früheren Epochen der Geschichte.

Das Gebiet ruft deshalb nach einem Handbuch, weil die internationale Forschung mittlerweile quantitativ wie qualitativ eine kritische Masse erreicht hat, die sie synthesebedürftig macht. Zugleich treffen in der Geschichtsschreibung über die Dekolonisation so viele unterschiedliche Perspektiven und Methoden zusammen, dass Vielfalt und Lebendigkeit gesichert sind. Das Spektrum reicht jenseits der kanonischen Politikthemen von Business History über Literatur und Film bis zu aktuellen

J. Osterhammel (四)

Freiburg i. Br., Deutschland

E-Mail: juergen.osterhammel@ frias.uni-freiburg.de 
Fragen wie dem Aufstieg eines humanitären Völkerrechts und dem Umgang mit den materiellen und moralischen Folgelasten des Kolonialismus.

Schließlich kommt es dem Handbook zugute, dass die wissenschaftliche Beschäftigung mit der Dekolonisation nicht durch große Kontroversen in Richtungen gespalten ist, wie das noch vor drei Jahrzehnten der Fall gewesen wäre. In der politischen Bewertung von Imperialismus, Kolonialismus und Dekolonisation sind sich die Verfasserinnen und Verfasser weitgehend einig. Zum Beispiel glaubt niemand mehr, dass Kolonien von großmütigen Regierungen ,in die Unabhängigkeit entlassen“" wurden. Gemeinsam ist den Beiträgen des Bandes eine genaue, aber nicht übertreibend heroisierende Aufmerksamkeit für Befreiungskämpfe und ihre jeweils besonderen regionalen Umstände. Ein Grundmotiv bildet auch der schnelle Umbruch des internationalen Systems und des moralischen Klimas in der Weltgemeinschaft. In weniger als einer Generation, schreibt Frederick Cooper, wandelten sich die Kolonialreiche ,from an ordinary fact of political life to the embodiment of illegitimate power" (S. 329).

Die Kapitel sind in vier Teile gruppiert. Unter der Überschrift „National Perspectives" findet man Skizzen von zehn imperialen Untergängen. Besondere Beachtung verdienen die ausgezeichneten Texte über die in der Literatur oft vernachlässigten Fälle Italien (Nicola Labanca), Portugal (Norrie MacQueen) und Japan (Louise Young): alle drei eher rasche Zusammenbrüche nach Krisen in den Metropolen als länger andauernde Selbstbefreiungen der Kolonisierten. Teil II (,Regional Perspectives") schaut auf Regionen in der kolonialen Welt und nimmt dabei häufig eine ungewohnt längerfristige Sichtweise ein. So lässt Christopher Goscha in seiner Diskussion Südostasiens die Umwälzung der Region bereits mit dem japanischen Angriff auf China 1937 beginnen. In derselben Weltgegend entdeckt Marieke Bloembergen ,international knowledge networks“ (S. 399), die Indonesien und Indien zu einem „Greater Indian mindset“ (S. 405) verbanden, dann aber mit dem indonesischen Nationalismus kollidierten.

Teil III („Thematic Perspectives“) versammelt regional übergreifende und vergleichende Beiträge. Neben unvermeidlichen Standardthemen - beispielsweise dem Kalten Krieg (Piero Gleijeses), Fluchtbewegungen und Fluchterfahrungen (Panikos Panayi) oder der Rolle von Gewalt und Gegengewalt (Martin Thomas) - findet sich eher Unerwartetes: David Motadel über islamische Widerstandsgruppen (deren Einfluss er aber nicht überschätzt sehen will) oder Miguel Bandeira Jerónimos' allzu knapp entwickelte These, die verschiedenen Ansätze zu spätkolonialer Entwicklungspolitik ließen sich einheitlich als ,repressive developmentalism“ (S. 537) deuten. Unter den sieben Kapiteln in Teil IV (,Legacies and Memories“) ragen Elizabeth Buettners pointierte, dabei aber statistisch recht dünn untermauerte Darstellung der nachkolonialen Migration nach Europa sowie Nicholas J. Whites meisterhafte Diskussion von spätkolonialem Kapitalismus und dem ,economic disengagement“ der Transitionsphase hervor. Es erstaunt, dass im ganzen Band sehr wenig von den Philippinen die Rede ist. Die spanische Westsahara fehlt ganz: immerhin ein postkolonialer Krisenherd. Das Thema ,,anti-colonialism as intellectual history“ hätte mehr verdient, als nur ein vierseitiger (und zwangsläufig oberflächlicher) Abschnitt in Christopher J. Lees' Kapitel über Anti-Kolonialismus zu sein. 
Im Vergleich zu anderen Oxford Handbooks legt das hier besprochene im Zweifelsfall den Akzent eher auf anspruchsvolle Forschungsdiskussion als auf elementare Einführung. Das macht es zu einem unentbehrlichen Hilfsmittel auch für Fortgeschrittene. Man sähe es gerne in jeder historischen oder sozialwissenschaftlichen Bibliothek, die kein Grundwerk zum 20. Jahrhundert verpassen will.

Funding Open Access funding provided by Projekt DEAL.

Open Access Dieser Artikel wird unter der Creative Commons Namensnennung 4.0 International Lizenz veröffentlicht, welche die Nutzung, Vervielfältigung, Bearbeitung, Verbreitung und Wiedergabe in jeglichem Medium und Format erlaubt, sofern Sie den/die ursprünglichen Autor(en) und die Quelle ordnungsgemäß nennen, einen Link zur Creative Commons Lizenz beifügen und angeben, ob Änderungen vorgenommen wurden.

Die in diesem Artikel enthaltenen Bilder und sonstiges Drittmaterial unterliegen ebenfalls der genannten Creative Commons Lizenz, sofern sich aus der Abbildungslegende nichts anderes ergibt. Sofern das betreffende Material nicht unter der genannten Creative Commons Lizenz steht und die betreffende Handlung nicht nach gesetzlichen Vorschriften erlaubt ist, ist für die oben aufgeführten Weiterverwendungen des Materials die Einwilligung des jeweiligen Rechteinhabers einzuholen.

Weitere Details zur Lizenz entnehmen Sie bitte der Lizenzinformation auf http://creativecommons.org/ licenses/by/4.0/deed.de.

\section{Bius, Joel R.: Smoke' Em if You Got'Em. The Rise and Fall of the Military Cigarette Ration, 328 S., Naval Institute Press, Annapolis 2018.}

\section{Mark Jakob}

Online publiziert: 23. Oktober 2019

(C) Gesellschaft zur wissenschaftlichen Förderung politischer Literatur e.V. and the Author(s) 2019

Joel R. Bius erkundet hier die Entstehung und den Niedergang der engen Beziehung zwischen der US-amerikanischen Zigarettenindustrie und der United States Army. Sein Untersuchungszeitraum erstreckt sich von der Einführung der kostenlosen Zigarettenrationen als Teil der Soldatenverpflegung im Ersten Weltkrieg bis zum Beschluss einer strikten Nichtraucherpolitik 1986. Bius' Darstellung schreitet nicht gleichmäßig chronologisch voran, sondern widmet die beiden Teile des Buches vorrangig dem Ersten Weltkrieg und der Zeit von 1964 bis 1986. Er will anhand

M. Jakob ( $₫)$

Marburg, Deutschland

E-Mail: mark.jakob@staff.uni-marburg.de 